\title{
La pisciculture au Cameroun: bilan et perspectives
}

\author{
Junie Albine ATANGANA KENFACK ${ }^{1 *}$, Christian DUCARME ${ }^{2}$ et \\ Jean-Claude MICHA ${ }^{3,4}$
}

\author{
${ }^{1}$ Département de Géographie, Université de Yaoundé I, Cameroun. \\ ${ }^{2}$ Piscimeuse, Belgique. \\ ${ }^{3}$ Unité de Recherche en Biologie Environnementale (URBE) Université de Namur (UN), Belgique. \\ ${ }^{4}$ Ecole Régionale Post-Universitaire d'Aménagement et de Gestion Intégrés des Forêts et Territoires \\ Tropicaux d'Aménagement (ERAIFT/UNESCO), Kinshasa, R.D. Congo. \\ *Auteur correspondant ; E-mail: juniealbine@yahoo.fr
}

\section{RESUME}

Introduite au Cameroun en 1948, la pisciculture a connu un succès qui s'est effondré après l'indépendance du pays. Pour tenter une analyse prospective du développement de l'aquaculture au Cameroun un bilan de la situation a été dressé et des perspectives ont été explorées. L'une des causes principales attribuées à cet échec fut la mise en œuvre de politiques inadaptées. L'élaboration de la stratégie nationale de l'aquaculture en 2003 avec un plan d'action précis en 2009 n'a pas suffi à relancer le sous-secteur de l'aquaculture. L'absence d'industries alimentaires pour poissons, l'accès difficile au crédit, l'absence et/ou l'insuffisance d'alevins de qualité, le manque de personnel qualifié, le manque de maîtrise des technologies de production et le mauvais aménagement d'étangs piscicoles sont autant de contraintes qui continuent de plomber le développement du sous-secteur. Pourtant le pays dispose de multiples atouts et des investissements importants sont réalisés en faveur du développement de la pisciculture. Pour l'avenir de la pisciculture au Cameroun, il faudrait sortir de l'amateurisme financier et recourir à une expertise certifiée, à du personnel compétent, motivé et bien payé.

(C) 2019 International Formulae Group. All rights reserved

Mots clés: Aménagements piscicoles, politique aquacole, Plan de développement de l'aquaculture, planification, stratégie aquacole.

\section{Fish farming in Cameroon: Balance and perspectives}

\begin{abstract}
Introduced in Cameroon in 1948, fish farming was a success that collapsed after the independence of the country. To try a prospective analysis of the development of aquaculture in Cameroon, a balance sheet on the situation was drawn up and the perspectives were explored. One of the main causes attributed to this failure was the implementation of inappropriate policies. The development of the national aquaculture strategy in 2003 with a specific action plan in 2009 was not enough to relaunch the aquaculture sub-sector. The lack of fish feed
\end{abstract}


industries, the poor access to credit, the lack and/or the insufficiency of quality fingerling, lack of trained personnel, lack of control over production technologies, and the development of fish ponds that are not adapted are all constraints that continue to limit the development of the sub-sector. Nevertheless, the country has many assets and important investments are made in favour of the development of fish farming. For the future of fish farming in Cameroun, we should exit from financial amateurism and resort to an expertise evidenced, to a qualified, motivated and well paid staff.

(C) 2019 International Formulae Group. All rights reserved

Keywords: Aquaculture policy, aquaculture development plan, aquaculture strategy, fish farm planning, planning.

\section{INTRODUCTION}

En 2015, selon la FAO, la production mondiale halieutique (poissons, mollusques et crustacés) a atteint un volume de 170 millions de tonnes $(94 \mathrm{Mt}$ pour la pêche et près de 76 Mt pour l'aquaculture dont $70 \%$ en eau douce et 30\% en eau de mer et saumâtre), auxquelles s'ajoutent environ $28 \mathrm{Mt}$ d'algues (FAO, 2016). L'organisation mondiale pour l'alimentation (FAO) estime qu'en 2025, la part de l'aquaculture atteindra $57 \%$ de la production halieutique soit une augmentation de $31 \mathrm{Mt}(+1,8 \%$ par an dans la prochaine décennie contre $+3 \%$ par an dans la précédente). Globalement, l'aquaculture constitue la production animale dont le développement est le plus rapide au niveau mondial (Micha, 2006 ; CESE, 2017).

Pourtant, au Cameroun, la production halieutique nationale n'est que de 180000 tonnes avec moins de 1000 tonnes/an provenant de l'aquaculture. Celle-ci reste faible pour une demande annuelle estimée à 400000 tonnes (ACP Fish 2011; MINEPIA, 2013). Pour combler ce déficit, 212000 tonnes de produits halieutiques ont été importés en 2012 (Isolina et al., 2013). La dépendance du pays à l'égard des importations devrait s'accentuer dans les années à venir, du fait de la constance de la demande et du recul de la production halieutique lié à la diminution des captures de poissons dans la plupart des zones de pêche.

En effet, comme dans de nombreux pays en développement, au Cameroun le poisson constitue la principale source de protéines, une véritable ressource de proximité que la viande ne peut remplacer du fait de son indisponibilité ou de son prix de vente élevé. C'est une source de protéines et d'oligoéléments essentiels, très précieuse pour l'équilibre nutritionnel et la santé (riche en oméga 3 à longues chaines et faible en cholestérol), qui représente environ $40 \%$ de l'apport protéique d'origine animale et 9,5\% des besoins totaux de la population (DSDSR, 2005 ; MINEPIA, 2009b). Actuellement, la consommation des produits de pêche avoisine $11 \mathrm{~kg} / \mathrm{habitant} / \mathrm{an}$ au Cameroun.

Par ailleurs, le pays possède un réseau hydrographique dense, comprenant plusieurs fleuves et rivières (3\% de la superficie des eaux continentales), des lacs naturels (4\%), des retenues de barrages (7\%), des plaines d'inondation et des marais ( $86 \%$ ), ainsi que de nombreux sites propices à l'aquaculture. Ce réseau hydrographique (Figure 1) offre un potentiel d'espèces piscicoles très diversifiées, avec 542 espèces de poissons sur plus de 40 $000 \mathrm{~km}^{2}$ de surface en eaux douces (MINEPAT, 2013).

A la différence de la Chine où la pisciculture est une activité vieille de près de 3 millénaires (Micha, 2013), la pisciculture a été introduite au Cameroun dans les régions du Centre et de l'Ouest vers 1948 par l'administration coloniale française (Satia, 1991 ; Tangou, 2009) comme technique de subsistance alimentaire avec un certain succès. Après l'indépendance, le sous-secteur s'est totalement effondré et la production piscicole a chuté totalement. Parmi les raisons évoquées figuraient une politique de développement inappropriée, l'absence de stratégie et de plans de développement du sous-secteur. 
Actuellement, le sous-secteur de la pisciculture est totalement sinistré, pourtant le pays dispose d'une politique nationale de la pêche et de l'aquaculture ainsi que d'une stratégie nationale de l'aquaculture depuis 2003 (MINEPIA, 2003) avec un plan d'action depuis 2009 (MINEPIA, 2009a).

Il apparaît donc que tous les éléments sont en place pour développer une aquaculture durable et rentable mais les difficultés persistent et le développement ne suit pas. L'objectif de cette étude est d'analyser le devenir de la pisciculture au Cameroun en vue d'identifier les meilleures options pouvant conduire au développement durable de l'activité. A cet effet, la revue des tentatives de développement de la pisciculture au Cameroun a été effectuée en vue d'aborder les perspectives pouvant conduire à un développement durable de l'activité.

\section{METHODOLOGIE}

La méthodologie adoptée dans cette étude repose sur la recherche documentaire et des entretiens avec les acteurs de la pisciculture. L'échantillonnage par grappe a permis de s'intéresser aux acteurs qui détiennent l'information pertinente dans le cadre de cette étude.

Les documents exploités ont été collectés auprès du Ministère de l'Elevage, des Pêches et Industries Animales (MINEPIA), du Ministère de l'Agriculture et du Développement Rural (MINADER), du Ministère de l'Economie, de la Planification et de l'Aménagement du Territoire (MINEPAT), dans les universités de Douala et de Dschang, à la FAO, de l'Institut National de Statistique (INS).

Des focus groupes ont été conduits avec 9 groupes de pisciculteurs répartis dans 3 des cinq régions (Centre, Est, Ouest) définies par le gouvernement comme ayant un fort potentiel aquacole au Cameroun, soit 3 groupes par région. Les guides d'entretien ont porté essentiellement sur les difficultés liées à l'exercice de la pisciculture, leurs attentes et les solutions proposées.

Par ailleurs, des interviews ont été réalisées auprès des responsables de l'aquaculture du MINEPIA (5), des responsables d'ONG locales (6) et des chercheurs du domaine de l'aquaculture (8). Les guides d'entretien ont porté essentiellement sur les difficultés liées à l'activité piscicole et les solutions préconisées.

Les données collectées ont été encodées à l'aide du logiciel Excel 2016 avant de faire l'objet d'une analyse des problèmes et d'une analyse du contexte.

\section{ETAT DES LIEUX DE LA PISCICULTURE AU CAMEROUN}

L'évolution de la pisciculture au Cameroun est tributaire de l'évolution de la politique économique nationale, du contexte économique de la sous-région et du contexte international.

\section{Entre 1960 et 1994 : les décennies de tentatives infructueuses}

L'administration coloniale française avec l'appui de l'Assemblée Représentative du Cameroun (ARCAM) avait tenté de promouvoir la culture du Tilapia en étangs de barrage dans les régions du Centre-Sud et de l'Ouest du pays entre 1948 et 1954. L'aquaculture était pratiquée uniquement en étangs et en eau douce (Satia, 1991). Pour cela, cinq (5) stations piscicoles avaient été installées. Il s'agit des stations aquacoles de Yaoundé, Foumban, Ngaoundéré, Mbouda et Bertoua. Malheureusement, ces projets n'ont pas connu de succès auprès des paysans, ceuxci n'ayant pas été associés dès le début des projets (Tangou, 2009) et ne s'étant pas appropriés la technologie.

Face à cet échec, la stratégie aquacole adoptée fut alors centrée sur la création des stations piscicoles ou centres d'alevinage, l'encadrement de la pisciculture rurale et la subvention d'équipement rural. C'est ainsi que vingt-deux (22) stations aquacoles ont été créées entre 1954 et 1960 et un service de vulgarisation a été lancé (FAO, 2004). La formation et l'encadrement des pisciculteurs étaient alors assurés par 130 moniteurs piscicoles environ et une trentaine de cadres expatriés du Corps de la Paix américain 
(Peace Corps) dont les activités portaient sur l'organisation des pisciculteurs, l'amélioration de la production d'alevins et la fabrication d'aliments pour poissons.

Parallèlement à la vulgarisation, les travaux de recherche menés dans les stations existantes permirent de sélectionner deux espèces de poissons ayant le double avantage de s'adapter aux conditions des étangs ruraux et d'être appréciées des consommateurs. Il s'agit des espèces Oreochromis niloticus et Heterotis niloticus. Cette fois, les stations ont fonctionné juste le temps des perfusions financières avant de tomber en ruine. Apparemment, l'approche de l'auto financement des stations n'aurait pas été développée. Ce fut un deuxième échec (Tangou, 2009 ; MINEPIA, 2009).

Entre 1961 et 1970, période qui correspond à la mise en ouvre des deux premiers plans quinquennaux, la pisciculture était destinée à compenser le déficit d'alimentation en protéines animales des populations des zones où l'élevage et la pêche n'assuraient pas de ressources suffisantes. Sa production n'était certes pas comparable à celle des pêches, mais sa portée restait considérable. L'objectif de la politique aquacole était donc triple :

- Porter au maximum la production des étangs par la vulgarisation des méthodes d'alimentation et la culture de nouvelles espèces ;

- Promouvoir la pisciculture partout où les conditions naturelles s'y prêtent ;

- Poursuivre les recherches scientifiques sur les meilleures méthodes d'élevage (premier plan quinquennal, 1960).

Cet art nouveau avait trouvé terrain fertile dans tout le Sud du territoire national jusqu'à Ngaoundéré. Notamment en raison des conditions naturelles favorables au développement de la pisciculture. Dès lors, la culture du tilapia en étangs de barrage était promue dans les régions du Centre et de l'Ouest du pays.

$\mathrm{Au}$ cours de cette période, le développement du Cameroun était amorcé depuis sa façade maritime longue de $400 \mathrm{~km}$ vers laquelle convergeaient les grands axes de trafic et d'échanges d'importance variable, plus ou moins ramifiés et desservant des terres d'inégale valeur, aux caractéristiques géographiques contrastées (FED, 1975). On distinguait ainsi trois grands groupes régionaux: le Sud-Cameroun, l'OuestCameroun et le Nord-Cameroun. Les grandes contraintes nationales (production et réseaux) et les potentialités physiques de ces grands groupes régionaux étaient alors identifiées afin d'orienter les actions de développement dans leurs aires d'influence. L'objectif était de profiter des avantages respectifs des différentes provinces du pays. Les zones à relief difficile recevaient les plus grandes subventions. Cette action avait pour avantage de faciliter la mise en place de projets régionaux.

C'est dans cette perspective que cinq zones au potentiel biophysique favorable au développement de la pisciculture avaient été sélectionnées. Il s'agissait de l'Adamaoua, du Centre, de l'Est, de l'Ouest et du Littoral. La période 1960-1994 a été marquée par l'absence d'un cadre légal et d'un document stratégique relatif à la filière aquacole. Toutefois, la pisciculture était une activité de subsistance et la production aquacole insuffisante. Dès les années 1971, avec la mise en œuvre du IIIe plan quinquennal, l'orientation générale de la politique aquacole visait les techniques piscicoles et l'alimentation des poissons. Les axes stratégiques reposaient alors sur la recherche scientifique (technique d'élevage, nouvelles espèces de poissons), la vulgarisation, l'identification des zones propices (en termes de potentiel physique, spatial et humain) et la formation. A la culture du Tilapia s'était ajouté Clarias gariepinus (= lazera) apporté de Bangui par J.-C. Micha (comm. Pers.) à Foumban dès 1971 dans le cadre du projet FAO de pisciculture régionale (RCA, Cameroun, Gabon et R. Congo). En 1969, l'on a introduit la carpe (Cyprinus carpio). La population piscicole s'était à nouveau agrandie en 1990 avec l'arrivée des carpes herbivores, Ctenopharyngodon idella (FAO, 2004).

Pour accompagner la réalisation des actions prévues dans la politique aquacole, le 
pays avait souscrit à plusieurs projets bilatéraux et à des interventions multiformes. En effet, entre 1968 et 1987, des organisations internationales (PNUD / FAO, FIDES, FAC, Peace Corps, OXFAM /SELF HELP, USAID, CRDI), la Banque Mondiale et même une société parapublique du Cameroun (SEMRY) ont œuvré chacune en son temps pour la redynamisation de l'activité piscicole avec des objectifs différents (ZAPI, 1987). Pendant que certains proposaient de vulgariser la culture du tilapia à travers la formation et ou l'encadrement des pisciculteurs, d'autres proposaient la culture de la carpe commune, la pisciculture intégrée à l'élevage de porcs et poules, la pisciculture associée à la culture du riz (dans le Nord du pays).

Entre 1991 et 1995, l'ONG américaine

Peace Corps avait proposé aux paysans un appui au développement de la monoculture de Tilapia. Il s'agit d'un mode d'élevage où l'on ne cultive qu'une seule espèce de poisson. La petite taille des poissons obtenus à la récolte avait découragé les paysans. Ce qui n'avait pas favorisé la reproduction des actions. La multiplicité des actions menées en faveur de la pisciculture n'avait pas suffi pour impulser le développement de l'activité. Les objectifs étaient rarement atteints. Pour preuve, que ce soit les techniques de reproduction des espèces cultivées ou l'encadrement, tous avaient disparu avec le projet. Même les étangs aménagés avaient été abandonnés à la fin des projets (Tangou, 2009). Bien que l'élevage et la pêche constituaient un secteur important de l'économie camerounaise, les activités et prérogatives des acteurs de l'aquaculture étaient limitées faute de lisibilité des marchés, ainsi que d'un cadre réglementaire clair. Ce manque de lisibilité de l'environnement institutionnel et les contraintes de l'environnement socioéconomique (accès limité aux ressources, aux intrants, aux financements et à la technologie) et physique (infrastructures rurales déficientes) auraient conduit la filière à une situation de stagnation voire de régression.
Entre 1994 et 2003 : vers une réorientation du rôle de l'administration dans l'aquaculture

Jusqu'au milieu des années 1980, le Cameroun avait inscrit sa politique de développement et ses choix d'investissement dans une vision stratégique à court terme, s'exprimant par le biais des plans quinquennaux de développement économique et social. Cependant, la crise économique de 1987 avait contraint le gouvernement à adopter une politique de gestion à très court terme. Cette situation a diminué la capacité d'intervention des pouvoirs publics dans les secteurs économiques et sociaux, contribuant ainsi à la modification du paysage des acteurs locaux de développement (Etamane, 2009). De fait, les structures étatiques jusque-là engagées dans la fourniture des services de base (santé, éducation, sécurité alimentaire, développement communautaire, etc.) ne purent continuer leurs missions, laissant ainsi un vide quelques fois comblé par les organisations de la société civile et le secteur privé.

Au cours des années 1996, la pauvreté avait fortement progressé au Cameroun et touchait plus de $50,5 \%$ de la population (BAD, 2002). De plus, l'offre publique de services avait été affectée par les difficultés financières de l'Etat qui étaient traduites par un indice de développement humain de 0,536. Cet indice classait le Cameroun au 134ème rang sur 174 pays en 1997. Aussi, la lutte contre la pauvreté était au centre des préoccupations de la politique économique, elle prévoyait l'adoption d'une stratégie de réduction de la pauvreté. Dans le secteur rural, la crise économique avait entraîné la baisse de la production agricole qui s'était aggravée avec la disparition des structures d'encadrement des agriculteurs (BAD, 2009). Dans ce contexte, l'approche développée dans la politique aquacole était moins interventionniste (MINEPIA, 2003), moins tournée vers l'atteinte de résultats quantifiés (augmentation du nombre d'étangs). Elle prenait davantage en considération les aspects de rentabilité et d'autonomie des systèmes de production, et consacrait le principe de la 
participation des promoteurs aux différentes étapes des projets.

En effet, les actions menées par le passé en faveur du développement de la pisciculture étaient le plus souvent des interventions non coordonnées, plutôt impulsées par les bailleurs de fonds (Tangou, 2009). Elles ne semblaient pas tenir compte des réalités du terrain et donc des paramètres socioéconomiques et culturels des zones d'intervention. C'est pourquoi les nouveaux projets de pisciculture avaient misé sur une démarche participative pour le décollage de la pisciculture au Cameroun (Tangou, 2009; MINEPIA, 2009b). C'est l'exemple du projet de recherche action développement de la pisciculture à Yemossoa (entre 1994 et 1997 avec le concours de la Coopération française) et du projet d'appui à la recherche participative en pisciculture et à la commercialisation des produits (entre 20002005 avec le World Fish Center). Le constat fut encore décevant, les rendements obtenus étaient faibles, les activités se sont arrêtées dès la fin des projets. Toutefois, la relance des activités du secteur agricole ne sera effective qu'après l'atteinte du point d'achèvement de l'Initiative Pays Pauvres Très Endettés (PPTE) intervenue en 2006.

\section{Depuis 2003 : aux origines de la mise en œuvre d'un cadre stratégique pour le développement durable de l'aquaculture}

En 2003, les politiques mises en perspective dans le Document Stratégique de Réduction de la Pauvreté (DSRP), traduisaient l'ambition du gouvernement d'œuvrer pour la réalisation des Objectifs du Millénaire pour le Développement (OMD). L'objectif de ces politiques était de réduire sensiblement la pauvreté au moyen d'une croissance économique forte et durable, d'une meilleure efficience des dépenses, de politiques de réduction de la pauvreté convenablement ciblées et du renforcement de la gouvernance (BAD, 2002 ; Etamane, 2009). Ainsi, la stratégie du gouvernement dans le secteur rural, visait à améliorer la productivité et les rendements agricoles. En ce qui concerne le secteur de l'élevage des pêches et des industries animales, la stratégie avait pour objectif de satisfaire les besoins des populations en protéines animales et d'améliorer le revenu des producteurs. C'est donc dans un contexte politique où les conditions macro-économiques ont imposé un changement radical de stratégie que l'idée de la mise sur pied d'un cadre stratégique pour le développement durable de l'aquaculture au Cameroun avait fait son apparition en 2003.

En effet, les nouvelles politiques exigeaient que le gouvernement cesse son rôle d'investisseur et de directeur d'entreprise pour adopter celui de facilitateur et de régulateur, le secteur privé étant le principal acteur du développement de l'économie du pays. Les objectifs stratégiques pour l'aquaculture étaient donc de satisfaire non seulement la demande locale, mais de contribuer à l'amélioration de la balance commerciale des produits aquacoles, de créer des opportunités d'emploi dans les zones urbaines et rurales tout en améliorant l'efficacité de la gestion des ressources naturelles (MINEPIA, 2009a). Les axes d'orientation de cette stratégie concernant l'identification des zones à fort potentiel aquacole, la définition des types d'aquaculture, la définition d'un cadre approprié de vulgarisation aquacole. C'est dans ce contexte, que le gouvernement avait tenté de céder des stations piscicoles aux acteurs du secteur privé (Tangou, 2009; WFC, 2011). On dénombrait environ 15000 étangs produisant 330 tonnes avec un rendement moyen de $400 \mathrm{~kg} / \mathrm{ha}$ (FAO, 2004).

Cependant, la mise en œuvre du DSRP, n'avait pas permis de réduire de manière significative la pauvreté au Cameroun. L'économie était demeurée fragile et entravée par des lacunes structurelles, relatives à la faible compétitivité du secteur productif (Mikolasek et al., 2009) et aux déficiences des facteurs de production clés que constituent les infrastructures et l'énergie. En conséquence, pour renforcer la reprise économique amorcée depuis une décennie et l'asseoir durablement, le gouvernement camerounais avait entrepris de réviser la stratégie de croissance économique et de réduction de la pauvreté en 2009. D'où l'élaboration du document de «vision de développement du Cameroun à l'horizon $2035 »$. La première décade de cette 
vision à long terme était couverte par la mise en œuvre du Document de Stratégie pour la Croissance et l'Emploi (DSCE) élaboré en 2009. Convaincu du rôle moteur du secteur rural dans la satisfaction non seulement des besoins alimentaires des populations, mais également des agro-industries, le gouvernement entendait procéder à la modernisation de l'appareil de production. L'accent était donc mis sur le développement d'hyper extensions agricoles dans les différentes régions du pays selon leurs spécificités agro écologiques afin de réaliser des rendements d'échelle et d'accroître substantiellement la production (DSCE, 2009).

Ainsi, dans le sous-secteur de l'élevage, des pêches et des industries animales, la stratégie restait ancrée au DSCE. Désormais, il ne s'agissait plus seulement d'autosuffisance alimentaire, mais aussi de commercialisation, de création d'emplois et de gestion des ressources naturelles. L'engagement du gouvernement dans cette nouvelle vision de développement s'était traduit entre autres en 2009 par l'élaboration du plan de développement durable de l'aquaculture qui mettait l'accent sur la commercialisation de la production. A long terme, l'impact positif du développement de l'aquaculture sur la réduction de la pauvreté serait perçu à travers la réduction du chômage des pauvres non qualifiés. Pour y arriver, la proposition du plan aquacole serait de créer au moins deux (2) emplois/ferme piscicole (MINEPIA, 2009a).

La dynamique observée dans le développement de la pisciculture s'était poursuivie avec l'implémentation de nouveaux projets piscicoles en vue de relancer l'activité. C'est le cas du Projet pour une Pisciculture Villageoise rentable dans les régions Centre et Ouest du Cameroun (PVCOC) qui avait tenté de vulgariser les techniques de production piscicole extensive (APDRA, 2011). Ce projet avait été mis en œuvre par le gouvernement avec l'aide de l'ONG française Association Pisciculture et Développement Rural en Afrique tropicale humide (APDRA) qui avait décidé de tester la pertinence au Cameroun d'un modèle piscicole paysan élaboré en Afrique de l'Ouest. Ce modèle consistait en l'élevage de poissons à base de la productivité naturelle des étangs de barrage pour le grossissement et de petits étangs de «service» pour la production d'alevins. Malgré la pertinence du modèle piscicole proposé, le développement de l'activité est resté limité. Les densités d'élevage étaient faibles $\left(0,5\right.$ Tilapia $\left./ \mathrm{m}^{2}\right)$.

Restant dans une optique de maintien de l'appui technique tel que suggéré dans la stratégie aquacole, le projet d'appui à la structuration des groupements et de la Profession avait été mis en œuvre (PSRPP) dans les mêmes zones, dans la période 20092011. Considérant que l'existence d'une organisation capable de défendre les intérêts de la profession, de garantir une production de qualité permettrait d'impulser une nouvelle dynamique à l'activité piscicole, ce projet avait pour objectif d'appuyer la création et la mise en réseau de groupes de pisciculteurs (APDRA, 2011). Dans la continuité de ses interventions au Cameroun, l'ONG APDRA et ses deux partenaires nationaux, le Service d'Etudes et d'Appui aux Populations à la Base (SEAPB) et le Centre d'Information, de Formation et de Recherche pour le Développement au Cameroun CIFORD), avaient mis en œuvre le Projet de Renforcement des Compétences des Pisciculteurs de la Région Centre (PRCP) entre 2011-2012. Le PRCP visait à renforcer les compétences des acteurs du secteur de la pisciculture paysanne (ONG partenaires, groupes de pisciculteurs) et à poursuivre l'accompagnement des producteurs en cours d'installation et en production (APDRA, 2013). Etant donné que la durée impartie à ce projet ne permettrait pas d'atteindre les objectifs fixés, deux projets conjoints avaient été mis en place dans la période 2013-2015 toujours dans le cadre du développement de la pisciculture. Il s'agit du Projet de Développement de la Pisciculture dans les régions Centre et Est du Cameroun (PDPCE) et du projet Systèmes Piscicoles Extensifs familiaux en Afrique de l'Ouest et Centrale (SyPiEx). Le PDPCE accompagnait les producteurs en cours d'installation et en production uniquement avec un appui 
technique (APDRA, 2013). Pendant que le projet SyPiEx proposait d'identifier et de comprendre les innovations piscicoles au niveau local afin de lever les principales contraintes au développement de l'activité. Apparemment, la durée des projets était courte (2 à 3 ans) et insuffisante pour garantir l'appropriation des techniques piscicoles par les pisciculteurs.

Sur le plan économique, la mise en œuvre du plan de développement de l'aquaculture devrait permettre de passer d'une production de 1000 tonnes à 8000 tonnes en 2015 puis à 80000 tonnes en 2020 . Cependant, la production aquacole actuelle demeure autour de 1.000 tonnes sur 155000 tonnes que représente la production halieutique nationale (FAO, 2013). Celle-ci est inférieure à la production réalisée dans les années $1980 \quad(3000$ à 4000 tonnes de poisson/an). Quant à la consommation de poisson, soit environ $9 \mathrm{~kg} / \mathrm{habitant} / \mathrm{an}$, elle est également inferieure à la valeur escomptée qui est de $11,57 \mathrm{~kg} / \mathrm{habitant} / \mathrm{an}$. Il est clair que le niveau de production a fortement chuté. Celleci ne satisfaisant pas la demande locale, elle ne pourrait donc améliorer la balance commerciale. Ce qui explique que les importations de poissons soient en hausse. De 8046 tonnes entre 1975 et 1976 , elles sont passées à 281450 tonnes environ en 2014 (INS, 2015). Ce qui ne cadre pas avec l'option d'autosuffisance alimentaire visé par le gouvernement. La sous-alimentation reste une réalité au Cameroun (DSDSR 2005). Sur 30 stations aquacoles mises en place depuis l'introduction de la pisciculture dans le pays, seules cinq (5) sont encore fonctionnelles (MINEPIA, 2009b). L'approvisionnement en alevins est essentiellement assuré par des écloseries privées. Toutefois, les prélèvements en milieu naturel constituent un autre mode d'approvisionnement en alevins. Quant à l'aliment industriel pour poisson, il est importé. Les productions artisanales locales sont de très mauvaise qualité tant nutritionnelle que qualitatives. Sa disponibilité est erratique. Leur qualité est souvent dégradée par une mauvaise conservation d'où la présence de mycotoxines. L'usine de production pour aliment construite à Bamenda n'est pas fonctionnelle, ses exigences en énergie électrique ayant été sous-estimées. Globalement l'approvisionnement en intrants est aléatoire et difficile, en raison de la défectuosité du réseau routier entre autres. L'essentiel de la production piscicole est realisée dans les systèmes de type extensif. Les systèmes semi-intensifs et intensifs sont rares (Mikolasek et al., 2009). Les systèmes d'élevage, sont des polycultures de Oreochromis niloticus et de Clarias gariepinus en association parfois avec Heterotis niloticus, Channa obscura (poisson à tête de serpent) et/ou Cyprinus carpio (Photo 1). Des systèmes d'intégration de l'élevage de porcs ou de poulets sont également pratiqués.

Toutefois, des capitaux importants sont injectés dans des projets visant la promotion des systèmes semi-intensifs des espèces Clarias gariepinus et tilapia (Oreochromis niloticus) dans des cages ou des bassins hors sol en béton et dans les cours d'eau (Photo 2). En 2014, le gouvernement a lancé la construction des centres de production intensive dans plusieurs régions du pays (Centre, Littoral, Sud, Sud-Ouest). Le projet d'«Appui au développement de l'élevage du tilapia en cage au Cameroun» consiste en la production locale des alevins, afin d'approvisionner les pisciculteurs du pays. Des centres de production intensive de poisson sont ainsi multipliés à travers des initiatives gouvernementales tel le programme Agropole (en 2010), le Programme de Promotion de l'Entreprenariat Agropastoral des Jeunes (PPEA-Jeunes en 2015), avec pour objectif de soutenir le développement d'entreprises des jeunes hommes et femmes (FIDA, 2016). L'utilisation d'aliments importés pour la production de tilapias et de poissons chat clarias, en système semi-intensif a fait l'objet d'une promotion importante dans le cadre du projet PPEA. La recherche est limitée aux expériences biotechnologiques et économiques. L'association des pisciculteurs aux activités de recherche n'est pas toujours effective. En matière de formation professionnelle, le pays dispose des établissements d'enseignement supérieurs (FASA de Dschang, ISH de Douala à 
Yabassi) où sont formés des ingénieurs en sciences halieutiques et d'un centre national de formation zootechnique et vétérinaire qui forme des techniciens de pêche et d'aquaculture (CNFZV de Foumban).

Les résultats de toutes ces actions de relance de la pisciculture ne sont pas toujours significatifs. De nombreuses contraintes persistent et concernent les institutions, les financements, les techniques d'aménagement et de gestion des sites, avec des effets sur la production et sur l'environnement.

\section{LES CONTRAINTES MAJEURES LIEES A L'EXERCICE DE LA PISCICULTURE}

Les contraintes piscicoles identifiées lors des entretiens dans les régions du Centre, de l'Est et de l'Ouest du Cameroun ont été catégorisées selon qu'elles relèvent des institutions, de l'aménagement, de la gestion ou du financement. Bien que les contraintes piscicoles soient nombreuses, certaines sont spécifiques à chaque région (Tableau 1) et appartiennent toujours aux classes définies dans ce cadre. Les résultats des analyses ont révélé que les contraintes piscicoles majeures sont généralement les mêmes pour les trois régions.

\section{Les contraintes institutionnelles}

Suite à l'examen approfondi des lois et règles $\mathrm{du}$ pays et d'après nos nombreuses observations sur le terrain, les contraintes institutionnelles (Tangou, 2009; MINEPIA, 2009b) concernent:

- La méconnaissance des lois et règles relatives à l'aquaculture. Les règles sont aussi nombreuses qu'étendues sur plusieurs secteurs d'activité;

- L'accès difficile au foncier, particulièrement dans les bas-fonds;

- L'application concrète des règles sur le terrain;

- La faiblesse de la communication relative aux activités du MINEPIA ;

- La méconnaissance des procédures à suivre pour obtenir une autorisation de création de ferme piscicole ;

- L'insuffisance d'encadrement par les agents de vulgarisation. Le mercantilisme de certains et la faible compétence technique de ces agents. Le système de vulgarisation est favorable à la pisciculture de subsistance alors que la politique promeut la pisciculture à orientation commerciale ;

- Les stations aquacoles sont mal gérées, avec des équipements obsolètes qui ne permettent pas de répondre efficacement aux besoins des pisciculteurs ;

- La disponibilité en alevins et en aliments n'est pas garantie. La densité moyenne de mise en charge des étangs reste faible $\left(0,5\right.$ alevins $/ \mathrm{m}^{2}$ pour tilapia par exemple). Les producteurs d'alevins de bonne qualité sont plutôt rares et concentrés dans la région du Centre, de l'Ouest et du Sud-Ouest. L'approvisionnement est d'autant plus difficile que l'accès aux lieux d'approvisionnement est difficile ;

- La mauvaise qualité des routes. L'accès aux fermes est difficile. Ce qui a des effets sur l'approvisionnement en intrants (fourniture d'alevins, nourriture, matières premières), la fréquence du suivi des élevages (tant par le pisciculteur que par les agents de vulgarisation), la distribution de la production et finalement sur le coût de production ;

- L'accès difficile aux alevins de bonne qualité. Le prix et la qualité des alevins ne sont pas fixés, ils dépendent des producteurs et souvent du rapport entre l'offre et la demande. Les tailles sont variables et les cohortes ne sont pas homogènes, la qualité sanitaire est entachée par de mauvaises manipulations et la difficulté du transport ;

- L'accès difficile à l'aliment de qualité. La valeur nutritive des aliments pour poisson n'est pas contrôlée. Les sousproduits utilisés ne font que rarement l'objet de test en laboratoire. Les conditions de conservation sont déficientes. Le prix de l'aliment importé n'est pas accessible aux promoteurs ;

- Le taux d'imposition trop élevé (45\%) sur les bénéfices des sociétés. Ce taux tend à renforcer le caractère informel de l'activité ; 
- Les sujets de recherche pas toujours coconstruits avec les promoteurs. Les relations entre les chercheurs et les promoteurs se limitent souvent aux enquêtes.

- Les bénéficiaires immédiats de la mise en œuvre de la politique aquacole sans tradition de l'aquaculture. Le temps d'appropriation des connaissances et techniques dans le domaine de l'aquaculture serait donc long. Il devrait être pris en compte dans la politique ;

- Le faible engagement de l'Etat dans la mise en place de partenariats entre les acteurs de la politique aquacole.

\section{Les contraintes liées à l'aménagement des étangs}

Toujours d'après nos constats sur le terrain et selon divers auteurs (Fongang, 2008 ; Mikolasek et al., 2009), ces contraintes comprennent :

- L'indisponibilité de la main d'œuvre qualifiée. Généralement ceux qui construisent les étangs ne sont pas spécialisés en ce type d'aménagements ;

- La faible sollicitation des services du MINEPIA. Les pisciculteurs semblent se limiter à leurs connaissances et leurs savoir-faire. Ils construisent de manière anarchique sans autorisation du MINEPIA ;

- Les exigences de prospection de sites sont jugées pénibles et coûteuses. Les pisciculteurs n'ont pas le temps ni les ressources financières nécessaires pour recourir aux services d'un topographe ou du MINEPIA ;

- L'insuffisance de moyens financiers. Selon les pisciculteurs, il faut disposer des ressources financières pour aménager le site, constituer le stock de poissons et gérer l'élevage ;

- Le caractère pénible des travaux manuels. En effet, il faut être physiquement fort et en bonne santé pour aménager et entretenir un site piscicole (Photo 3). Les pisciculteurs dont la moyenne d'âge est de +/- 50 ans estiment ne plus avoir assez de force pour ces travaux ;
- La présence de vase dans les étangs ou leur envasement progressif (Photo 4). Non seulement la récolte n'est pas aisée et la vase est délétère pour la qualité de la récolte et, de plus, elle favorise la croissance des plantes aquatiques dans l'étang ;

- L'inadéquation des étangs piscicoles avec le milieu biophysique. L'emplacement des étangs dans les basfonds, leur profondeur, leur accessibilité et leur source d'approvisionnement en eau (Photo 5) ne favorisent pas toujours de bonnes conditions de gestion de l'eau et des déchets ;

- Les conflits d'usage des terres. Ils sont liés aux chevauchements des activités notamment entre agriculture et élevage ou entre habitation et élevage. En général la sécurité foncière n'est pas garantie.

\section{Les contraintes liées à la gestion des étangs}

D'après nos observations de terrain appuyées par les documents de divers auteurs (Mikolasek et al., 2009, Mikolasek et Oswald, 2013), les contraintes relatives à la gestion des étangs sont :

- La proximité des étangs piscicoles. Les effluents des étangs situés en amont entrainent l'inondation des étangs situés en aval et la perte de poisson ;

- Les inondations. En saison des pluies, les étangs reçoivent les eaux de ruissellement et les eaux usées en provenance des activités anthropiques. Le type d'aménagement ne favorise pas le contrôle de l'eau (moines, surverses) ;

- La rupture des digues. Les inondations provoquent souvent la rupture des digues occasionnant ainsi la perte des alevins ou des poissons présents dans les étangs. Celles-ci ne sont pas toujours suffisamment solides pour supporter la pression de l'eau ;

- La pollution des cours d'eaux. Les pécheurs utilisent souvent les pesticides pour pêcher dans les cours d'eau qui alimentent les étangs piscicoles. De plus la qualité et le volume des déchets d'ordures drainés par la pluie ou déversés par les populations ne sont pas 
contrôlés. Pourtant la pisciculture est très sensible à la qualité de l'eau et à ses changements ;

- La prolifération des plantes aquatiques. Les plantes aquatiques envahissent la surface et les bordures des étangs. Celles-ci abritent souvent des prédateurs tels que les serpents, varans et des fourmis qui constituent des dangers pour le pisciculteur lors de l'entretien des étangs. Sans entretien du plan d'eau, ces plantes peuvent entrainer son comblement progressif (atterrissement) ;

- La profondeur des étangs qui dépasse parfois deux mètres. Elle atteint dans certains cas $4 \mathrm{~m}$. Dans ce cas, l'énergie solaire n'atteint pas le fond du bassin. De fait la productivité de l'eau est réduite ;

- A l'inverse, une profondeur inférieure à un mètre, ce qui est souvent le cas, limite la production de l'étang ;

- La faible organisation des associations piscicoles. La démarche piscicole est individuelle. Chacun fait comme il veut, ou comme il peut. Les intérêts sont défendus individuellement ;

- Le manque de qualification des groupes piscicoles. Les pisciculteurs ne sont pas des professionnels de l'aquaculture. Cependant les plus anciens ont un certain savoir-faire ;

- Les systèmes de vidange sont parfois insuffisants. Les canaux d'évacuation des eaux sont de petite taille et souvent cèdent à la pression de l'eau (photo 6) ;

- La prédation des poissons. Pendant que les grenouilles consomment les alevins, les oiseaux consomment les poissons des étangs ;

- Les rendements sont faibles. Les pisciculteurs désirent connaitre des techniques modernes d'élevage afin d'améliorer leurs rendements.

\section{Les contraintes économiques}

D'après nos diverses enquêtes et selon divers auteurs (Tangou, 2009; MINEPIA, 2013), elles portent sur :

\footnotetext{
- L'absence de crédit à un taux acceptable ;
}

- Le faible engagement des structures bancaires et micro finances dans la mise en œuvre de la politique aquacole. Elles ne sont pas suffisamment sensibilisées sur l'activité aquacole ;

- Les faibles compétences managériales des promoteurs piscicoles. Leurs projets sont souvent mal montés, incomplets pour attirer l'attention des banques. Ils ne tiennent pas souvent de fiche de gestion des étangs ;

- La mauvaise organisation du marché des intrants et des produits de la pisciculture. Les marchés de référence pour la vente de poisson d'élevage, d'alevins, ou d'aliment pour poisson sont plutôt rares.

Ces contraintes expliquent que la pisciculture ne se soit pas développée et soulèvent les besoins de formation et d'encadrement des pisciculteurs, de disponibilité et d'accès aux intrants et aux sites; autant d'activités qui doivent être réalisées à long terme par l'Etat afin de conduire le développement d'une activité piscicole durable.

\section{Enjeux et Perspectives}

La plupart des problèmes en aquaculture proviennent du manque d'une complète compréhension des éléments essentiels devant être pris en compte dans les processus de sélection et de gestion des sites. Des décisions basées sur des informations incomplètes et des politiques inadaptées peuvent compromettre le développement durable de l'aquaculture (Hishamunda et al., 2011). Une des stratégies pour optimiser la durabilité de l'activité piscicole au Cameroun serait basée sur la sélection et la gestion des sites piscicoles, qui font partie des éléments les plus importants pour la réussite de l'activité (UICN, 2009). Pour ce faire, des connaissances du marché, juridiques, environnementales, techniques et socioéconomiques fiables sont nécessaires.

Sur le plan des marchés, les aquaculteurs n'ont aucune connaissance des marchés réels ni des possibilités pour obtenir un meilleur prix de leur production. Leurs projets piscicoles ne mettent pas l'accent sur la faisabilité économique. De plus, la 
production des matières premières fourragères (soja, tournesol, maïs, etc.) importante dans la composition de l'aliment pour poisson en Afrique est insuffisante pour approvisionner les marchés locaux. Les sites piscicoles restent éloignés des marchés, ce qui ajoute un coût de logistique. Sachant que le marché est la base du développement d'une activité économique durable, l'organisation de la profession pourrait contribuer à une meilleure commercialisation des produits piscicoles. Toutefois, le besoin de professionnalisation de la filière est prégnant pour tous les maillons de la chaîne des valeurs, partant des producteurs d'alevins et d'aliment pour poisson, des fournisseurs de services, des producteurs au consommateur.

Actuellement, les pisciculteurs ne seraient pas encore capables de fournir du poisson d'élevage de qualité de taille adéquate, en temps et lieu requis par les acheteurs et à prix compétitif avec celui offert par la pêche ou les poissonneries (importation) tout en ayant une marge bénéficiaire. Leur capacité à faire face à la concurrence reste faible. Vraisemblablement, à l'échelle nationale, la dotation en ressources, la technologie, la productivité, les caractéristiques du produit semblent être les facteurs les plus importants qui conditionnent la compétitivité de l'aquaculture. Il est important de recadrer le développement de l'aquaculture dans le contexte de concurrence permanente de tous les produits carnés, notamment le poisson congelé, le bœuf, le porc et la volaille (Lacroix et Paquotte, 1999).

Sur le plan juridique, les zones idéales pour les aménagements piscicoles appartiennent au domaine public fluvial. Les biens du domaine public sont insusceptibles d'appropriation privée. La politique aquacole n'a pas encore réglé cette question foncière. L'élaboration d'un cadre légal spécifique à l'aquaculture permettrait donc de garantir la sécurité foncière des pisciculteurs. Les acteurs de la pisciculture devraient avoir une connaissance claire de la législation régissant l'activité et des règles de planification correspondantes. Dans ce but, le pays devrait disposer d'une législation transparente en matière d'aquaculture afin de fournir aux aquaculteurs une sécurité suffisante du point de vue juridique.

D'un point de vue administratif. Des outils visant à dynamiser ou à améliorer les pratiques piscicoles (guide pour l'aquaculture, etc.) d'une part, et, des dispositifs d'accompagnement (financier, technique et administratif) d'autre part pour les porteurs de projets piscicoles devraient être mis en place au niveau local, régional et national avec la participation de tous les acteurs. Un lien étroit devrait être établi entre ces outils et ceux dédiés aux autres pratiques agricoles. La cohérence entre les administrations publiques et au sein du ministère en charge de l'aquaculture permettrait à l'aquaculture de se faire une place aux côtés des autres activités de productions alimentaires au sein du territoire.

En effet, les fermes aquacoles sont par nature fortement dépendantes du milieu dans lequel elles sont installées, ce qui crée des interactions fortes entre elles et l'agriculture, comme avec les autres activités du territoire. $\mathrm{Au}$ Cameroun, la pêche et l'aquaculture en eau douce relèvent d'un même ministère. De plus, les pisciculteurs s'approvisionnent souvent en alevins auprès des pécheurs. Ces interactions obligent une certaine collaboration entre les pisciculteurs et les pêcheurs. En mutualisant leurs forces, ces acteurs pourraient mieux défendre leurs intérêts communs.

En ce qui concerne l'organisation de la filière aquacole, elle est éclatée entre de nombreuses structures et interprofessions : les ONG locales, et internationales (œuvrant dans la pisciculture, dans l'agriculture ou dans le domaine agricole, voire social), les fabricants d'aliments, les menuisiers (fabriquant de dispositif de vidange), les pisciculteurs et les revendeurs de poisson. Une fédération de l'aquaculture permettrait de mieux organiser la filière sur l'ensemble du territoire.

Sur le plan environnemental, le site reconnu devant la loi devrait satisfaire les exigences biophysiques requises pour le bon développement des poissons (bonne qualité de l'eau et du sol). Pour éviter que les étangs ne soient abandonnés, ceux-ci devront être aménagés sur des sites adaptés. Les conditions 
environnementales et le système de production devront également être en adéquation pour s'assurer que les infrastructures piscicoles intègrent leur environnement (FAO, 2011). Ainsi l'évaluation des exigences biophysiques de l'activité permettra de délimiter la zone à exploiter tout en tenant compte des besoins en espace pour l'extension de la ferme.

En effet, le développement souhaitable de la pisciculture au Cameroun impliquera nécessairement une augmentation des espaces consacrés à cette activité. En raison de l'agrandissement des fermes existantes et/ou la création de nouvelles. Il est nécessaire de penser à une organisation territoriale de cette activité pour corriger son développement anarchique. La disponibilité des zones adaptées à la pisciculture devient un problème important pour le développement et la croissance de cette activité au regard des pressions sur les ressources naturelles et de la récurrence des conflits d'usage de la terre et de l'eau. Selon une étude récente, l'infrastructure géodésique, est caractérisée par l'absence d'un référentiel géodésique fiable et unique, ce qui complexifie la situation foncière, voire l'affectation des terres au Cameroun (Tchawa, 2014). Environ $5 \%$ de personnes vivant en milieu rural dispose d'un titre foncier. Une bonne connaissance de ce référentiel géodésique permettrait de mieux organiser l'espace à l'échelle nationale et partant de là, une meilleure identification des sites piscicoles potentiels. Dans la pratique, la sélection de sites aquacoles repose très souvent sur une vaste gamme de facteurs incluant les critères géologiques, physico-chimiques, biologiques et écologiques, la demande locale (Little et al., 2013), la proximité des moyens de transport et de marchés porteurs. Le choix d'un bon site pour un étang piscicole est l'un des facteurs les plus importants pour déterminer sa faisabilité économique, minimiser les interactions avec l'environnement et concilier sa présence avec d'autres activités (MARM, 2011).

De même, la pisciculture est tout aussi fortement dépendante, pour sa qualité et sa quantité, des autres usages en amont et en aval des bassins versants. Il est donc nécessaire d'anticiper le partage des ressources entre les différents usages de l'eau dans les territoires et bassins versants. Ces interactions justifient la nécessité d'un niveau élevé d'encadrement juridique et la cohérence de la politique aquacole avec les autres politiques publiques.

Toutefois, le développement des infrastructures de base serait nécessaire pour soutenir la croissance de la production piscicole. En effet, le développement des fermes générera une hausse proportionnelle des quantités d'aliments pour poisson qui sont souvent produits en dehors de la région dans laquelle se trouve la ferme. La création d'une provenderie pour poisson devrait tenir compte de la distance entre la ferme et le site de production de l'aliment. La maîtrise d'une alimentation de proximité et de qualité est donc un enjeu majeur pour les fermes piscicoles. On doit se poser la question de la disponibilité (spatio temporelle), de la qualité et du coût des matières pour produire cet aliment. De plus les provendiers manquent de trésorerie. La disponibilité de courant $(380 \mathrm{~V})$ à fort ampérage est indispensable pour ce type d'installation. C'est ailleurs la raison pour laquelle l'usine construite par l'Etat n'est pas fonctionnelle (MINEPAT, 2016).

Sur le plan technique, il est important de connaître le système de production piscicole adapté aux caractéristiques du milieu afin de mieux gérer les risques de l'activité. Ces connaissances techniques impliquent également le choix des espèces à cultiver et du matériel à utiliser pour la réussite du projet. Les résultats zootechniques (indice de conversion) et le prix élevé de l'aliment (1200 à $1500 \mathrm{CFA} / \mathrm{kg}$ ) sont incompatibles avec une rentabilité économique de l'élevage du tilapia. Dans le cas du clarias, au vu de son prix de vente plus élevé (2000 à $2500 \mathrm{CFA} / \mathrm{kg}$ contre 1500 à $1800 \mathrm{CFA} / \mathrm{kg}$ pour le tilapia) et des indices de conversion faibles $(0,8$ à 1 pour les meilleures piscicultures), des productions rentables sont envisageables.

$\mathrm{La}$ pisciculture extensive est généralement pratiquée dans des étangs de barrage. Ceux-ci sont difficiles à gérer (Adjanke et al., 2016) parce que les différents paramètres de production ne sont pas 
maîtrisables et qu'il est presque impossible de les vidanger complètement. De plus, leur production décroît après la première récolte (MINEPIA, 2013). Toutefois, il est possible d'améliorer le niveau de production et de rentabilité avec les ressources disponibles actuellement, en adoptant des techniques d'élevage appropriées (Olubunmi et al., 2015 ; Dada, 2015). En outre, il est possible de minimiser la majeure partie des impacts potentiels de l'aquaculture à condition de connaître les processus mis en œuvre, de pratiquer une gestion responsable et de déterminer correctement l'emplacement des installations d'aquaculture (UICN, 2007).

D'une manière générale, les recherches devraient être menées continuellement afin d'améliorer les connaissances en aquaculture. Celles-ci devront être disponibles et compréhensibles par tous. Le développement de la pisciculture au Cameroun devrait reposer sur l'élevage des espèces Oreochromis niloticus et Clarias gariepinus, qui sont endémiques à 1'Afrique. Oreochromis niloticus, dont la production annuelle mondiale (2017) approche les 5 millions de tonnes, reste une des meilleures espèces mondiales pour l'aquaculture, bien connue et parfaitement maitrisée. Le pays devrait sélectionner des souches à croissance rapide et passer à l'élevage mono sexe mâle à partir d'alevins de même âge et de qualité certifiée provenant éventuellement d'un sexage manuel, d'une inversion de sexe par méthyl testostérone ou mieux de la technique plus récente des super mâles YY (Micha, 2013). Compte tenu des compétences requises pour la reproduction artificielle et l'alevinage de ces espèces de poisson, une formation spécifique des techniciens et des fermiers est nécessaire.

Cependant, cette technologie exige des connaissances et des compétences approfondies, et par conséquent des formations adaptées, tant pour les professionnels que pour les fonctionnaires, sur la conception et la mise en œuvre des projets piscicoles, sur les aménagements piscicoles et sur les techniques d'élevage.

D'un point de vue socio-économique, l'accès aux financements dans la pisciculture reste une contrainte majeure. Actuellement au Cameroun, il n'existe pas de fonds de garantie pour l'aquaculture, ni de système de prêts bonifiés auprès des institutions bancaires. Toutefois, les taux bonifiés varient entre 1,5 à $2 \%$ par mois ce qui est supérieur au taux de rentabilité d'une pisciculture. Dans ces conditions, ce n'est pas imaginable de recourir à ces emprunts. L'absence de garantie sur la terre constitue un des facteurs qui limite l'accès au crédit. L'Etat devrait sensibiliser les institutions bancaires sur la rentabilité de la pisciculture, en s'appuyant notamment sur des business plans élaborés, le suivi des fermes pilotes, et la mise en place d'un système fiable de statistiques et d'informations sur l'activité.

De même, le taux d'imposition sur le bénéfice de $45 \%$ appliqué à la pisciculture serait un autre facteur qui pousse les opérateurs à travailler dans l'informel sans avoir recours au MINEPIA ou autres structures d'appuis. Généralement, les pisciculteurs ne déclarent pas leur activité par crainte de devoir payer des taxes. Une révision de la valeur de ce taux dans le cadre de la mise en œuvre de la politique aquacole serait un avantage pour les promoteurs.

Il convient de noter que la prise en compte d'un seul de ces facteurs ne suffirait pas à impulser le développement de l'activité piscicole. Loin d'être une équation facile, le développement de la pisciculture nécessite une batterie de conditions sociales, économiques, politiques, environnementales et culturelles afin de s'assurer que celle-ci s'intègre à l'économie locale et que les sites soient sélectionnés et gérés de manière concertée et adaptée à l'environnement.

Il ressort de cette analyse que les choix politiques effectués dans le cadre du développement de l'aquaculture au Cameroun n'ont pas permis d'atteindre les objectifs fixés. La production nationale aquacole stagne autour de 1000 tonnes/an alors que la demande en poisson ne cesse de croître depuis l'indépendance du pays. Les étangs piscicoles sont relativement petits et difficilement vidangés de manière complète. Ils sont situés dans des zones enclavées, souvent éloignés des marchés. Les techniques de production sont rudimentaires et les intrants de bonne 
qualité ne sont pas toujours disponibles, voire accessibles. Les performances de la politique aquacole ne seraient pas suffisantes pour impulser le développement durable de l'activité aquacole. L'aquaculture demeure soumise à des contraintes administratives, financières, techniques, de marketing et socioéconomiques qui entravent le développement durable du sous-secteur. L'augmentation de la production aquacole sera fonction :

- De l'élaboration d'un cadre légal et réglementaire approprié prenant en considération les contraintes et potentialités de l'activité et définissant clairement les droits fonciers des pisciculteurs. Les pays ayant connu une forte augmentation de leur production, comme le Chili et l'Espagne, ont une loi spécifique à l'aquaculture. Selon la FAO, les exigences légales d'une licence et d'une évaluation d'impacts sur l'environnement avant la création d'un étang piscicole justifieraient les progrès obtenus dans la maîtrise de la gestion de l'eau dans les fermes piscicoles, au Ghana et dans l'amélioration de la production ;

- De l'adoption d'une approche globale et intégrée aux niveaux stratégique et local. Plusieurs études font états des interactions entre les composantes économiques, sociale, environnementale et institutionnelle de l'aquaculture. En effet, les mesures à concevoir pour développer l'aquaculture doivent considérer à la fois les implications sociale, environnementale et économique des modifications envisagées dans la conduite de l'activité. La sélection des sites appropriés devra tenir compte de la proximité avec les marchés, des conditions environnementales et socioéconomiques du milieu (UICN, 2009). De même, la fertilisation des étangs à partir des systèmes de production intégrés (Montchowui et al., 2012; Mikolasek et Oswald, 2013) à l'agriculture (rizi-pisciculture, maraîchage-pisciculture, etc.) ou à l'élevage (volaille, porcs, lapins, etc.), comme c'est le cas en Asie, sont à privilégier. Toutefois ceux-ci doivent être respectueux de l'environnement ;

- De la cohérence entre les politiques publiques. Une politique aquacole claire, concertée et partagée, en harmonie avec les politiques des finances, des cadastres, des travaux publics, de l'agriculture, de la gestion de l'eau, etc. favoriserait l'efficacité et l'efficience des interventions relatives au developpement de l'aquaculture. Des mesures incitatives telles que la réduction du taux d'imposition, la mise en place de fonds pour l'aquaculture favoriseraient le développement de l'activité. La recherche devrait proposer une gamme variée d'espèces d'élevage. Notamment les espèces à haute valeur ajoutée : poisson à tête de serpent (Parachanna), sole du fleuve (Schilbe), crevette, daurade, bar, etc. (Micha, 2006). En chine, l'introduction d'espèces nouvelles à haute valeur commerciale a stimulé le développement de grosses entreprises de production d'aliments (FAO, 2000).

\section{SYNTHESE}

A l'évidence, le Cameroun, comme beaucoup d'autres pays africains, voit ses ressources halieutiques décliner par surexploitation, entre autre, alors que sa consommation de poisson ne fait que croître tout comme sa population qui de plus tend à s'orienter vers la consommation de poisson plutôt que vers la viande, comme source de protéines animales. Etant donné ces constats, le pays a tenté depuis son indépendance et tente toujours, comme la plupart des autres pays du continent, de développer le secteur de l'aquaculture dont les perspectives sont bonnes vu la demande nationale croissante en produits halieutiques. Un tel secteur avec une filière bien organisée peut rapidement contribuer à un fort développement durable d'une région (delta du Mékong au Vietnam, delta du Nil en Egypte, périphérie de Lagos au Nigeria, etc.). Et pourtant au Cameroun, on n'enregistre pas de franc succès, on ne repère aucune région où la pisciculture, la crevetticulture, etc. connaissent un essor spectaculaire rentable et durable. Les espèces 
utilisées, la plupart endémiques à l'Afrique (Oreochriomis niloticus, Clarias gariepinnus, etc..), sont pourtant bien connues et leur élevage parfaitement maitrisé dans le monde entier, notamment en ce qui concerne le tilapia (Oreochromis spp.), produit actuellement à près de 5 millions de tonnes par an. Pourquoi donc, malgré les bonnes perspectives, le Cameroun ainsi que beaucoup d'autres pays africains, surtout francophones (R Congo, RD Congo, Gabon, etc..), ne parviennent pas actuellement à faire décoller la pisciculture chez eux. Est-ce que le prix de vente du poisson à la pisciculture reste trop faible pour assurer un revenu suffisant à l'exploitant? Au Vietnam, le prix de vente du tilapia vivant à la sortie de la pisciculture est de l'ordre de $1 € / \mathrm{kg}$ ce qui assure la rentabilité de l'exploitation. Le prix de vente au détail du tilapia au Cameroun étant de l'ordre de 1500 à $1800 \mathrm{CFA} / \mathrm{kg}( \pm 2,75 €)$, la rentabilité devrait aussi être assurée, à moins que l'ensemble des coûts de production et de l'amortissement ne soient plus élevés au vu de l'ensemble des éléments évoqués dans: «Enjeux et Perspectives ». Mais alors la production de poisson chat (Clarias gariepinus) avec un prix de vente au détail de 2000 à $2500 \mathrm{CFA} / \mathrm{kg}$ devrait parfaitement couvrir tous ces coûts et sa production rentable devrait décoller.

Pour un plein essor futur de la pisciculture, faut-il compter sur des appuis publics tel que pratiqués depuis des décennies en Afrique et au Cameroun en particulier («Décennies de tentatives infructueuses») ou plutôt compter sur des investissements privés basés sur des motivations personnelles de pisciculteurs nouveaux ? La plupart des Etats africains et le Cameroun en particulier n'ont pas su gérer correctement leurs propres stations de pisciculture implantées en différentes régions pour y servir de moteur de l'activité. Il faut plutôt attendre de l'Etat la constitution d'un cadre favorable à cette nouvelle activité dont certains acteurs privés motivés et de préférence personnellement impliqués vont tirer parti pour développer une pisciculture durable et rentable. En d'autres termes, le rôle de la planification au niveau national et régional dans le développement du secteur est très important sans pour cela que les instances étatiques soient impliquées dans l'investissement. Que l'Etat joue son rôle régalien en sécurisant les investisseurs dans un cadre défini par lui.

Mais, les promoteurs piscicoles doivent également mettre tous les moyens nécessaires au bon développement de leur nouvelle activité. On constate trop souvent que des privés ayant quelques finances en réserve, espérant des revenus rapides, importants et constants, se lancent dans ce secteur sans y mettre tous les moyens nécessaires, ce qui ne peut aboutir favorablement et durablement. Tout «business plan» en pisciculture, qui devient actuellement légion, ne donnera de résultats probants que si la conception technique du système de production est parfaitement élaborée. Trop souvent de grands projets de production de poissons sont mal ou incomplètement conçus par des investisseurs tentant de limiter leurs dépenses dans des projets pourtant couteux, faits de bric et de brocs pour ne pas recourir à une expertise valable mais plus coûteuse. Comme dernier exemple vécu, une pisciculture de tilapia en « raceway » est construite pour produire 360 t/an en utilisant l'eau de nappe souterraine pompée à $80 \mathrm{~m}$ de profondeur, stockée dans un château d'eau de $500 \mathrm{~m}^{3}$ et cela sans tour de dégazage. Dès la mise en production, les maladies, telle que la maladie des bulles (embolie gazeuze) se sont manifestées.

Pour l'avenir de la pisciculture en Afrique et au Cameroun, il faudrait sortir de cet amateurisme financier trop fréquent et les investisseurs ne doivent pas oublier que la croissance du poisson prend du temps et que les premiers revenus de l'investissement ne seront perceptibles que près de 2 ans plus tard, à condition de recourir à une expertise certifiée, à du personnel compétent, motivé et bien payé. La pisciculture est donc soumise à des contraintes administratives, financières, socio-économiques, techniques et de «marketing» qui mal on incomplètement prises en compte impacteront son développement et sa durabilité. 
Tableau 1: Contraintes piscicoles spécifiques aux régions du Centre de l'Est et de l'Ouest (Source: enquêtes de terrain, 2013).

\begin{tabular}{|c|c|c|}
\hline Région Centre & Région Est & Région Ouest \\
\hline Absence de Main d'œuvre & Litige fonciers & Relief accidenté \\
\hline $\begin{array}{l}\text { Coût de location engin } \\
\text { lourd élevé }\end{array}$ & Spéculation sur le foncier & $\begin{array}{l}\text { Pente des collines } \\
\text { menant aux sites abrupte }\end{array}$ \\
\hline Conflits au sein des groupes & $\begin{array}{l}\text { Rupture des digues fréquente. } \\
\text { Prolifération des plantes envahissantes, } \\
\text { des sangsues. } \\
\text { Vols lors de la récolte, prédation } \\
\text { Profondeur des étangs importante } \\
\text { (environ } 3 \mathrm{~m})\end{array}$ & \\
\hline
\end{tabular}

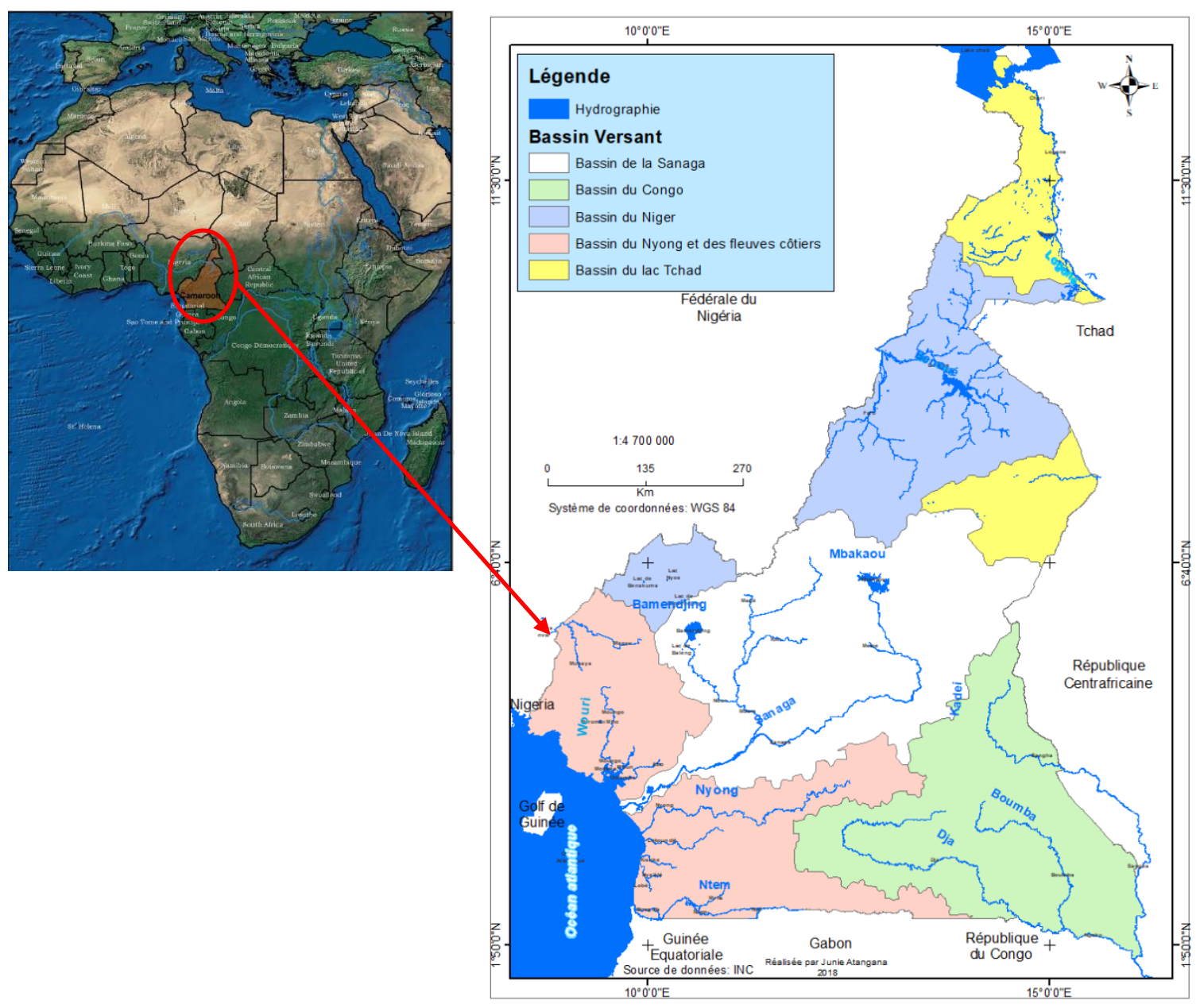

Figure 1: Réseau hydrographique du Cameroun (Olivry, 1986). 


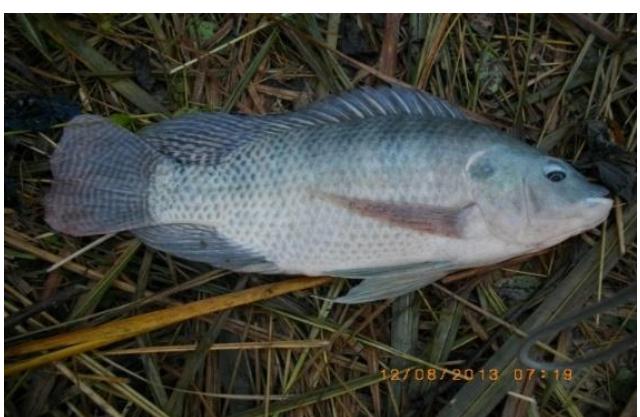

Oreochromis niloticus

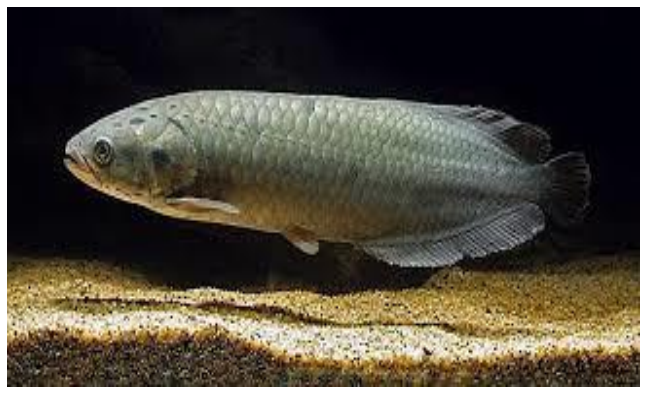

Heterotis niloticus

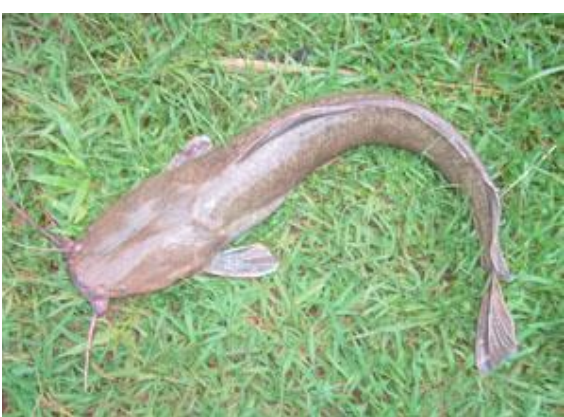

Clarias gariepinus

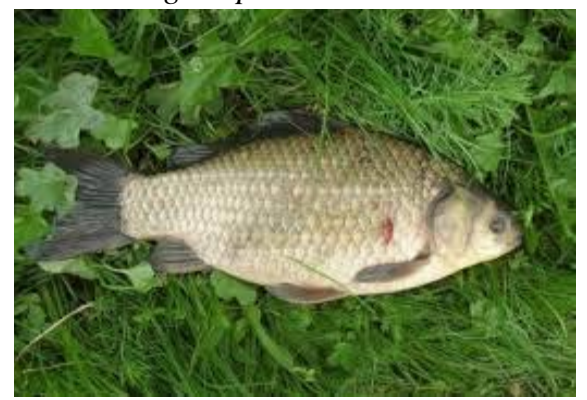

Cyprinus carpio

Photo 1 : Espèces de poissons élevées au Cameroun.

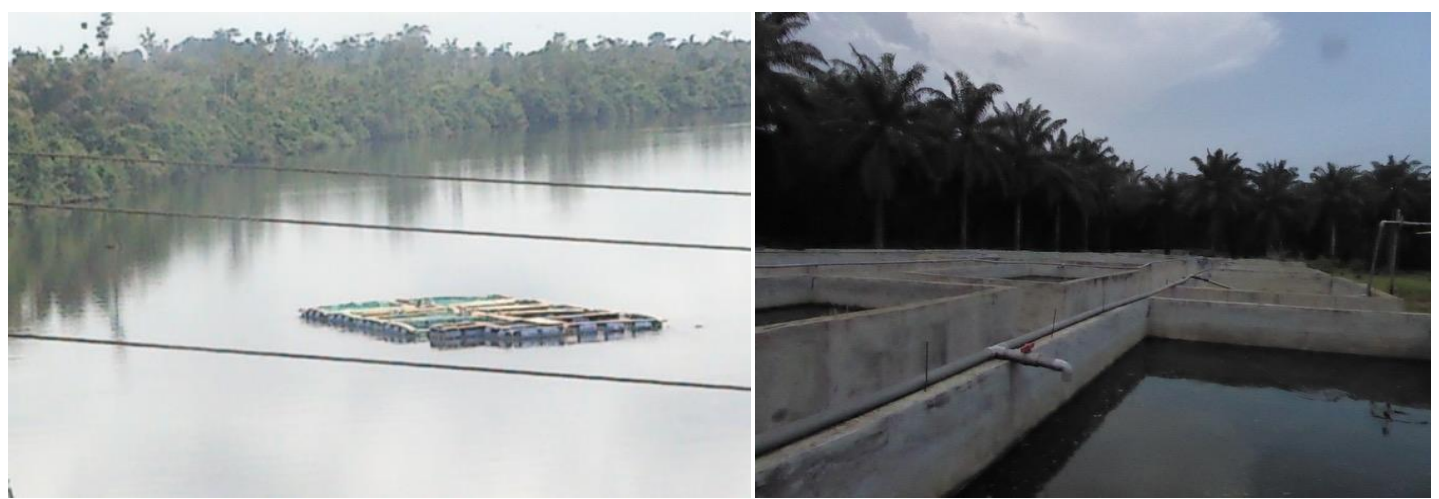

Photo 2 : Pisciculture dans les rives d'un cours d'eau (Littoral) et dans des bassins en béton (Sud).
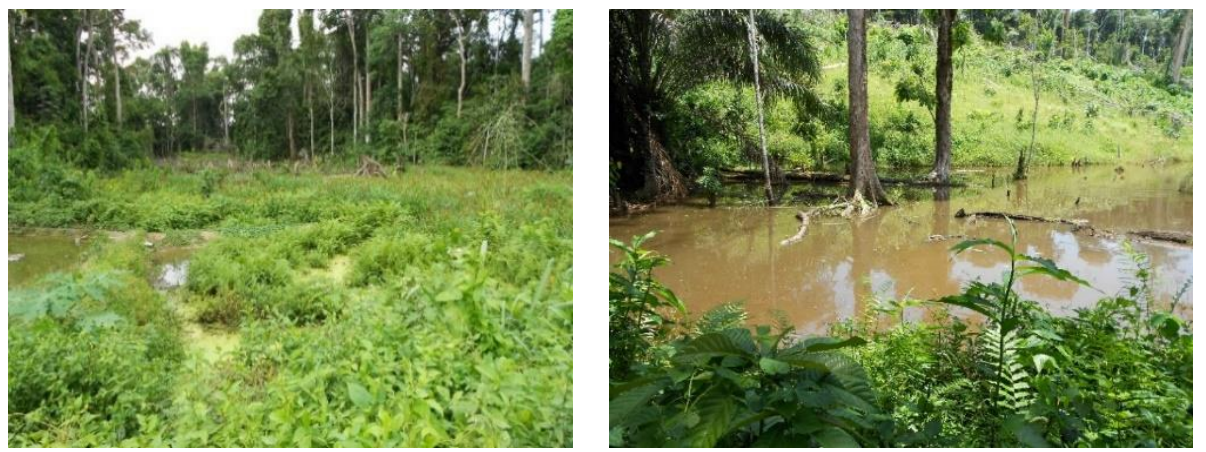

Photo 3 : Etat des étangs piscicoles dans la région du Centre (Cameroun). 

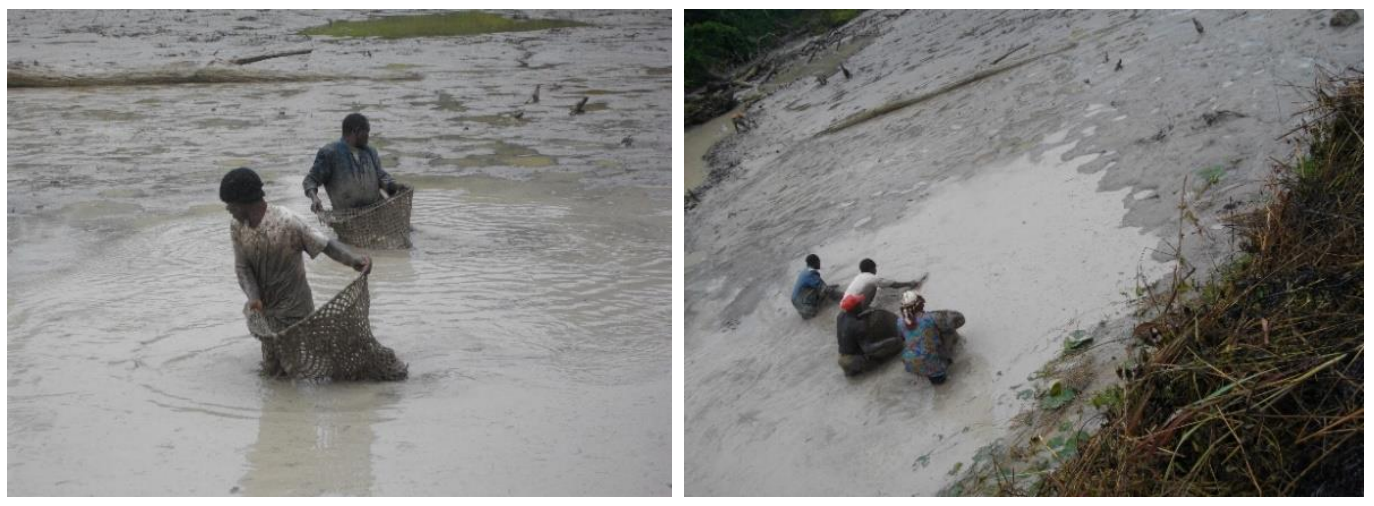

Photo 4 : Etangs envasés (région de l’Est du Cameroun).
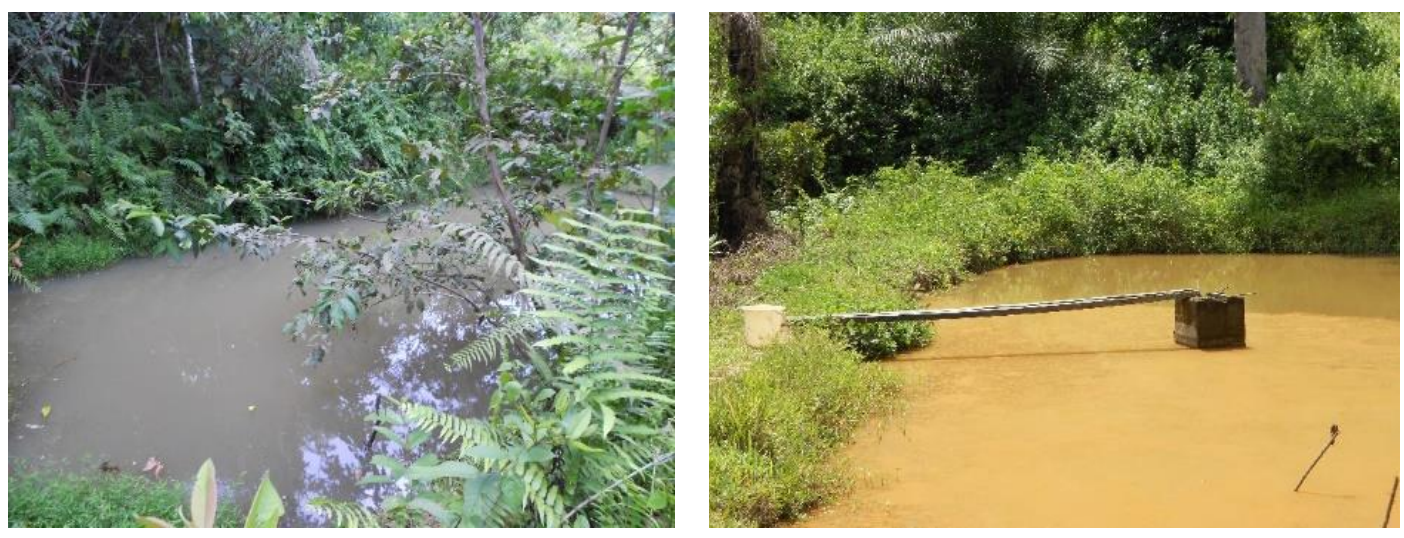

Photo 5 : Etangs de nappe phréatique dans la région du Centre.
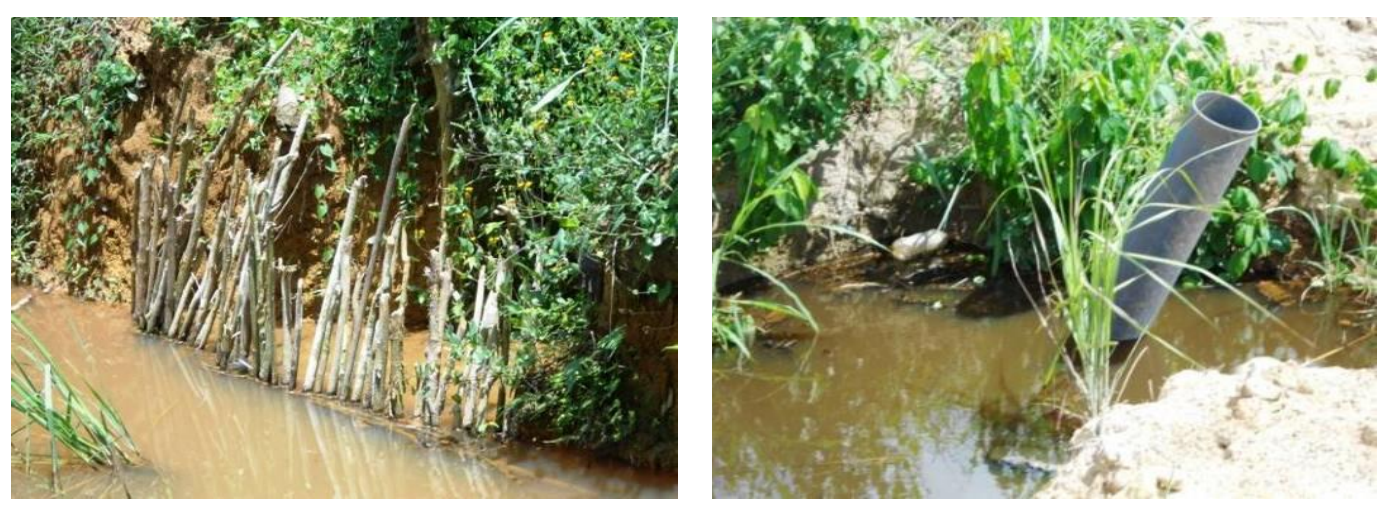

Photo 6 : Systèmes de vidange (déversoir en bambou dans la région du Centre et en tuyau PVC dans la région de l'Est). 


\section{Conclusion}

L'analyse historique de la pisciculture au Cameroun a fait état d'un développement lent et erratique qui nécessite un engagement fort des pouvoirs publics et des collectivités pour garantir le développement durable de cette activité dans le pays. A l'évidence, l'élaboration des instruments de pilotage de la politique aquacole n'a pas suffi à relancer le sous-secteur. La pratique d'une pisciculture de subsistance, le maintien de l'insécurité foncière, le développement anarchique des aménagements piscicoles inadaptés, le manque de connaissances et de compétences spécifiques à la pisciculture, la globalisation des différents types de pisciculture, l'incohérence entre les politiques publiques successives, sont autant d'éléments qui montrent l'inadéquation des choix politiques effectués dans le cadre du développement de l'aquaculture. Qu'il s'agisse des finances, ou de la nature, les ressources sont limitées et les changements-rapides. Il est nécessaire d'opérer des choix cohérents et de planifier correctement afin d'utiliser les ressources de manière plus efficace et efficiente. En effet, de par son caractère singulier, la pisciculture nécessite des ressources et une combinaison unique de conditions sociales, économiques, environnementales et de gouvernance pour un développement durable. Le rôle de la planification au niveau national et régional dans le développement du secteur est très important sans pour cela que les instances étatiques soient impliquées dans l'investissement. Il faudrait également recourir à une expertise certifiée, à du personnel compétent, motivé et bien payé. En tout cas, le Cameroun, comme d'autres pays africains, n'auront bientôt plus le choix, vu leur croissance démographique et l'extinction en cours de leurs stocks de poissons pêchés, il faudra bien trouver les voies et moyens de développer une aquaculture rentable et durable comme l'ont fait la plupart des pays des autres continents et même comme commencent à le faire les pays africains anglophones.

\section{CONTRIBUTION DES AUTEURS}

JAAK, auteur principal, a été impliquée dans toutes les phases de cette étude, la collecte, l'analyse et l'interprétation des données, la structuration et la rédaction de l'article. CD et JCM ont contribué à l'analyse, l'interprétation et la correction de l'article.

\section{REMERCIEMENTS}

Les auteurs remercient toutes les personnes interviewées dans le cadre de cette étude pour leur disponibilité. Ils remercient également Monsieur SOULEY Bonaventure Olivier pour sa contribution financière à la production de cet article.

\section{CONFLIT D'INTERETS}

Les auteurs déclarent n'avoir aucun conflit d'intérêts.

\section{REFERENCES}

ACPFish II. 2011. Structuration des moyens intra-institutionnels (privés et publics) et de relations inter-institutionnelles aux niveaux national et international dans la filière crevetticole au Cameroun. Projet $\mathrm{n}^{\circ} \mathrm{cu} / \mathrm{pe} 1 / \mathrm{gb} / 10 / 005.190 \mathrm{p}$.

Adjanke A, Tona K, Agbohessi PT, Toko II, Gbeassor M. 2016. Current situation of fish farming in Togo. Int. J. Biol. Chem. Sci., $\quad$ 10(5): 2015-2024. https://www.ajol.info/index.php/ijbcs/art icle/viewFile/153691/143279.

APDRA. 2011. Projet de Structuration d'un Réseau Pérenne de Pisciculteurs dans les régions Centre et Ouest du Cameroun PSRPP. FSD 2008-004205. 54 p.

APDRA. 2013. Projet de renforcement des compétences des pisciculteurs de la région Centre du Cameroun PRCP. Rapport final. 21 p. www, apdra.org

BAD. 2002. Cameroun: Programme d'ajustement structurel II (PAS II). Rapport d'évaluation de performance de projet (REPP). Département de l'évaluation des opérations (OPEV). 14 novembre 2002. 55p.

BAD. 2009. Document De Stratégie Pays 2010-2014. Banque Africaine De Développement Fonds Africain De Développement. Département Régional Centre (ORCE). $42 \mathrm{p}$.

CESE. 2017. Les fermes aquacoles marines et continentales : enjeux et conditions d'un développement durable réussi. Avis du 
Conseil Economique, Social et Environnemental. Section de l'agriculture, de la pêche et de l'alimentation. 98 p. ww.lecese.fr

DADA Adekunle Ayokanmi. 2015. Use of fluted pumpkin (Telfairia occidentalis) leaf powder as feed additive in African catfish (Clarias gariepinus) fingerlings. Int. J. Biol. Chem. Sci., 9(1): 301-307. http://ajol.info/index.php/ijbcs/article/11 8842/328537.

DSDSR. 2005. Document stratégie de développement du secteur rural, $189 \mathrm{p}$.

Etamane MAT. 2009. Les grandes orientations des politiques économiques au Cameroun sous l'ère du président Ahmadou Ahidjo de 1960 à 1982: analyse historique. Analele Universităţii, Dunărea de Jos" Galaţi, seria 19, Istorie, VIII : 213-227.

FAO. 2000. Développement de l'aquacculture en Chine; rôle des politiques gouvernementales. http://www.fao.org

FAO. 2004. Vue générale du secteur aquacole national.

http://www.fao.org/fishery/countrysector /naso-cameroon/fr

FAO. 2011. Directives techniques pour une pêche responsable. Le développement de l'aquaculture : une approche écosystémique de l'aquaculture. Supplément $4.76 \mathrm{p}$.

FAO. 2016. Situation Mondiale des Pêches. Possibilités et Défis. FAO ; 275 p.

FED. 1975. Cameroun 1960-1975. Fond Européen pour le Développement. Délégation de la commission des communautés européennes. Centre de documentation.

Division « développement et coopération». Bruxelles. S.A Van In, Lier. 55p.

FIDA. 2016. Programme de Promotion de l'Entreprenariat Agropastoral des Jeunes. Rapport de Supervision 05 au 19 avril 2016. Rapport principal et appendices. Division Afrique de l'Ouest et du Centre Département de Gestion des Programmes. Rapport : 4095-CM. 55p.

Fongang D. 2008. Identification des sources potentielles d'apports en éléments organiques et minéraux dans les étangs piscicoles : cas de Bertoua (Est-
Cameroun). Mémoire de fin d'étude FASA, Dschang. 98p.

Hishamunda N, Poulain F, Ridler N. 2011. Analyse prospective du développement de l'aquaculture. La méthode Delphi. FAO, document technique sur les pêches et l'aquaculture, 521. 89p.

INS. 2015. Chapitre 15: Elevage et Pêche. In Annuaire Statistique du Cameroun. INS, Edition 2015 ; P257-268.

Isolina B, Suzanne P, Mariaeleonora D'Andrea. 2013. Pisciculture : le nouveau moteur de l'économie bleue? Briefings $\mathrm{N}^{0} 32$ de Bruxelles sur le développement rural Une série de réunions sur des questions de développement ACP-U. 51p.

Lacroix D, Paquotte P. 1999. L'aquaculture et les marchés : tendances, produits, opportunités. Journées aquacoles de l'Océan Indien - Ile de la Réunion 31 mai-3 juin 1999. 15p.

Little DC, Murray FJ, Leschen W, Waley D. 2013. Socio-economic factors affecting aquaculture site selection and carrying capacity. In Site Selection and Carrying Capacities for Inland and Coastal Aquaculture. Ross LG, Telfer TC, Falconer L, Soto D, Aguilar-Manjarrez J (eds). FAO proceedings $\mathrm{N}^{\mathrm{o}} 21 ; 103-115$.

MARM. 2011. Aquaculture continentale et environnement. Ministère de l'Environnement et du Milieu Rural et Marin Secrétariat Général Technique Centre de Publications. 51p.

Micha J-C. 2006. Pas d'avenir sans pisciculture : le big bang piscicole. 20p.

Micha J-C. 2013. La pisciculture dans le bassin du Congo : passé, présent et futur. USTHB-FBS-4th International Congress of the Populations \& Animal Communities "Dynamics \& Biodiversity of the terrestrial \& aquatic Ecosystems""CIPCA4"TAGHIT (Bechar) - Algeria, 19-21 November, 2013. p 85-101.

Mikolasek O, Barlet B, Chia E, Pouomogne V, Tomedi Eyango Tabi M. 2009. Développement de la petite pisciculture marchande au Cameroun : la rechercheaction en partenariat. Cah Agric, 18(2-3). 270-276. DOI : 10.1684/agr.2009.0277 
Mikolasek O, Oswald M. 2013. Intensification écologique de la pisciculture paysanne. Exemple du Cameroun. Démarche et cadre conceptuel. 62p.

MINEPIA. 2003. Cadre stratégique de l'aquaculture durable au Cameroun. 16p.

MINEPIA. 2009a. Plan de développement de l'aquaculture durable au Cameroun. 62p.

MINEPIA. 2009b. Revue sectorielle du secteur aquaculture. Mise en place d'un plan de développement durable de l'aquaculture au Cameroun. 44p.

MINEPIA. 2013. Recensement des fermes piscicoles dans les zones à fort potentiel au Cameroun (Centre, Est, Ouest, NordOuest et Sud). Rapport principal. 32 p.

MINEPAT. 2013. Diagnostic sur la situation de référence du Cameroun. Vol 1. 246p.

MINEPAT. 2016. Elaboration du schéma national d'aménagement et de développement Durable du territoire du Cameroun (SNADDT). Rapport de diagnostic. Secteurs économiques et dynamiques spatiales. 481p.

Montchowui E, Agadjihouede H, N'tcha E, Laleye P. 2012. Effets de milieux d'élevage sur la survie et la croissance des juvéniles de la carpe africaine, Labeo parvus Boulenger, 1902. Int. J. Biol. Chem. Sci., 6(5): 2131-2138. DOI: http://dx.doi.org/10.4314/ijbcs.v6i5.20

Olivry JC. 1986. Fleuves et Rivières du Cameroun. Collection Monographies Hydrologiques ORSTOM D No 9: Paris; $781 \mathrm{p}$.

Olubunmi TA, Imogie GM, Shofela S. 2015. Protein profile of breeding discrepancies of African catfish Clarias gariepinus in aquaculture practices. Int. J. Biol. Chem.
Sci.,

9(5):

2648-2653.

https://www.ajol.info/index.php/ijbcs/art icle/viewFile/130165/119734

Satia NBP. 1991. Historique du développement de la pisciculture au Cameroun.

Tangou S. 2009. Evaluation des réglementations et des programmes aquacoles au Cameroun. Projet SARNISSA en 2009. 44 p. www.sarnissa.org.

Tchawa P. 2014. Amélioration de la gouvernance du secteur foncier au Cameroun. Mise en œuvre du Cadre d'Analyse de la Gouvernance Foncière. Rapport du Cadre de l'Analyse de la Gouvernance Foncière au Cameroun, The world Bank. 119 p.

UICN. 2007. Interactions entre l'Aquaculture et l'Environnement. Guide pour le Développement Durable de l'Aquaculture en Méditerranée. UICN ; $118 \mathrm{p}$.

UICN. 2009. Guide pour le Développement Durable de l'Aquaculture Méditerranéenne 2. Aquaculture: Sélection et Gestion des Sites. UICN. VIII: Gland, Suisse et Malaga, Espagne ; $340 \mathrm{p}$.

WFC. 2011. Aquaculture, Fisheries, Poverty and Food Security. WFC; 62p. http://pubs.iclarm.net/resource_centre/W F_2971.pdf.

ZAPI. 1987. Integrated rural development project. Project completion report Cameroon. Western Africa Projects Department Agriculture D. CREDIT 776-CM. Report No. 6808. 61p. 